Document downloaded from:

http://hdl.handle.net/10251/102632

This paper must be cited as:

Zambrano-Vizuete, OM.; Pérez Llopis, I.; Carvajal Rodrigo, FJ.; Esteve Domingo, M.; Palau Salvador, CE. (2017). Command and Control Information Systems Applied to Large Forest Fires Response. IEEE Latin America Transactions. 15(9):1735-1741. doi:10.1109/TLA.2017.8015080

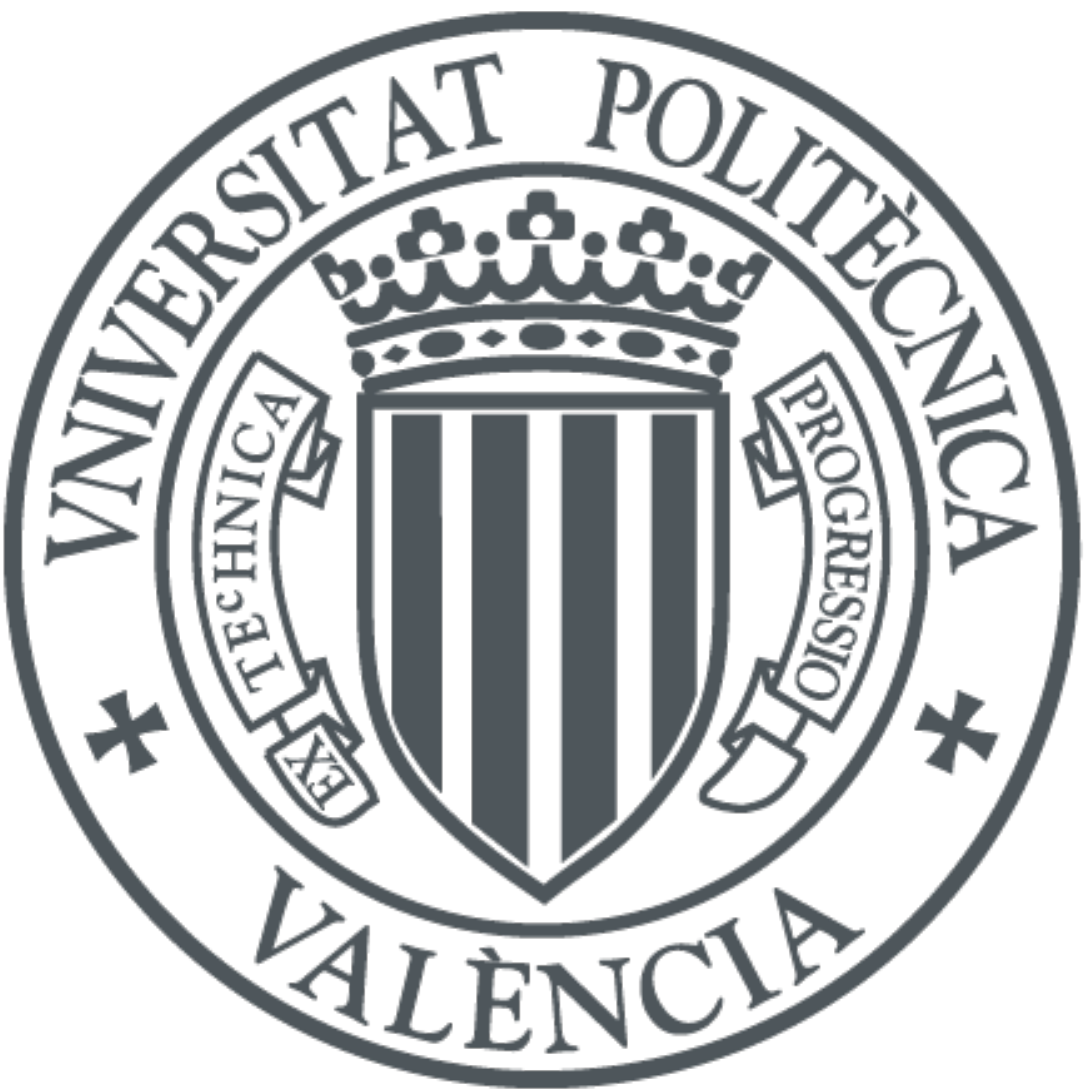

The final publication is available at

https://doi.org/10.1109/TLA.2017.8015080

Copyright Institute of Electrical and Electronics Engineers

Additional Information 


\title{
Command and Control Information Systems Applied to Large Forest Fires Response
}

\author{
M. Zambrano, I Pérez, F. Carvajal, M. Esteve and C. Palau
}

\begin{abstract}
Forest fires are one of the natural disasters that frequently occur around the world, causing irreparable human, material and environmental losses. It is essential to have an accurate description of the operations environment to confront a wild forest fire. This fact allows taking decisions in line with reality and collaboration among the different agencies involved in the response operations. This paper describes an architecture for implementing of a Command and Control Information System that facilitates to obtain an accurate situational awareness of the operations scene, communications with the deployed units inside and outside the disaster environment, and coordination and effective transmission of decisions making when responding to the presence of a wild forest fire. It focus its proposal on the establishment of a tactical network that allows adequate monitoring of the environment and facilitate mobility and deployment of response units on the hot spot.
\end{abstract}

Keywords - Command and Control Information System, Decision Making, Large Forest Fire, Situation Awareness, Wireless Mesh Network.

\section{INTRODUCCIÓN}

$\mathrm{D}$ ESDE SU APARICIÓN, el hombre ha tenido que coexistir con diferentes tipos de desastres naturales, los cuales han ido dejando a su paso importantes secuelas en los núcleos sociales en los que se han presentado. Uno de los tipos de desastres naturales más frecuentes y destructivos son los Grandes Incendios Forestales (GIF), causados en un 95\% de los casos por la actividad humana, ya sea de forma accidental o intencional, han tomado relevancia en esta última década debido a su constante incremento y a las grandes pérdidas materiales, ambientales y humanas que provocan [1] [2] [3]. Entre las principales características de los GIF, se pueden mencionar las de afectar grandes extensiones territoriales (abarcando en la mayoría de los casos diversas jurisdicciones y superando las barreras urbano forestales), su velocidad de expansión, dificultad de extinción, y el alto riesgo al que están expuestos los brigadistas en las operaciones de respuesta [4] [5].

Para enfrentar un GIF de manera efectiva, es imperativo contar con una Conciencia Situacional (CS) que describa claramente el entorno del desastre y permita a todos los involucrados tomar decisiones y acciones adecuadas, oportunas y acordes con la realidad. La CS es un factor crítico en cualquier actividad en la que la complejidad y dinámica del

M. Zambrano, Universitat Politècnica de València, Valencia, España, oszamvi@ doctor.upv.es

I. Pérez, Universitat Politècnica de València, Valencia, España, ispello@upvnet.upv.es

F. Carvajal, Universitat Politècnica de València, Valencia, España, fecarrod@upvnet.upv.es entorno, afecte negativamente la toma de decisiones. Puede definirse de manera general, como la representación mental y la comprensión del entorno que influye sobre las decisiones y acciones humanas, o dicho de otra manera, conocer y entender lo que está ocurriendo, para saber lo que se debe hacer [6] [7]. La CS está directamente relacionada con la calidad de la información (precisión, inteligibilidad y disponibilidad); por un lado, respecto a la fidelidad con la que puede representar el entorno de operaciones, y por otro, respecto a la oportunidad y disponibilidad que ofrece a sus usuarios. La producción y circulación de una información de calidad al momento de enfrentar un desastre natural como en el caso de los GIF, contribuye a eliminar la incertidumbre y generar confianza en el Sistema de Mando y Control (C2S, por sus siglas en inglés) [8], lo que a su vez, favorece la aceptación de las decisiones adoptadas, la correcta ejecución de las mismas, y un trabajo coordinado y colaborativo entre las fuerzas de respuesta de todas las agencias participantes [9].

Los Sistemas de Información y Comunicaciones (SIC) son los responsables de gestionar la información dentro de una organización, garantizando su calidad y distribución en todo momento y lugar [9] [10]. Los SIC son el soporte principal de los $\mathrm{C} 2 \mathrm{~S}$, y dentro del ámbito de la gestión de emergencias y desastres, por lo general, se los conoce como Sistemas de Información para Mando y Control (C2IS, por sus siglas en inglés).

Este artículo presenta una arquitectura para la implementación de un C2IS, que permita conformar una CS precisa y oportuna del entorno de operaciones al momento de enfrentar un GIF. Hace énfasis sobre la geolocalización, el vídeo, y las capacidades de las Redes Inalámbricas Malladas (WMN, por sus siglas en ingles), como factores clave para la obtención de la información que permita una adecuada descripción del entorno, la supervisión de los recursos desplegados dentro del mismo (sensores, equipos, vehículos, personal de respuesta, etc.), y la distribución de esa información durante las operaciones de respuesta.

La validación de la propuesta, se ha realizado por medio de pruebas de funcionalidad a un prototipo implementado en base a la arquitectura descrita en este artículo, el cual ha permitido la identificación y seguimiento de dos brigadistas desplegados dentro del escenario simulado para un GIF, y la transmisión en tiempo real de los vídeos provenientes de las videocámaras de cada uno de ellos.

M. Esteve, Universitat Politècnica de València, Valencia, España, mesteve@dcom.upv.es

C. Palau, Universitat Politècnica de València, Valencia, España, cpalau@dcom.upv.es 


\section{MOTIVACIÓN}

Una de las características más importantes de un desastre natural es la agresividad de su entorno, y particularmente en el caso de los GIF, esta agresividad tiene especial relevancia debido a los riesgos que se presentan en la zona de afectación durante las operaciones de respuesta. Incendios como el de Alberta (Canadá, 2016), Victoria (Australia, 2015), Islas Canarias (España, 2007), Quillón (Chile, 2012), son muestras del poder destructivo del fuego y la importancia del conocimiento del entorno de operaciones al momento de responder [10]. Los avances tecnológicos y los progresos en la gestión de emergencias y desastres realizados en estos últimos años, han permitido mejorar considerablemente las tareas de respuesta al momento de enfrentar los GIF, con brigadistas mejor preparados y con recursos materiales y técnicos que brindan un mayor apoyo y disminuyen el riesgo de brigadistas y damnificados [7]. Una respuesta efectiva, tiene sus raíces en una CS precisa, oportuna y común entre todos los involucrados, y para ésto, es necesario contar con un C2IS que permita obtener y distribuir la información requerida, en el momento requerido.

El principal aporte de este trabajo, está en el desarrollo de una arquitectura para la implementación de un C2IS, que permita la adquisición de una CS real del entorno del GIF, y cree las bases para los procesos de mando y control, coordinación y colaboración entre las fuerzas de respuesta involucradas.

\section{ARQUITECTURA}

Se fundamenta en la efectiva supervisión del entorno de operaciones y las capacidades de las WMN [11] [12], como medios para obtener y distribuir la información necesaria para conformar una CS precisa y oportuna del escenario del GIF. Ha sido pensada para dar soporte a los siguientes requerimientos:

(i) Sensorización: debe permitir la integración de diferentes tipos de sensores que entreguen información referente a las condiciones del medio ambiente de operaciones (temperatura, humedad, velocidad y dirección del viento, etc.), y el estado de las unidades y personal de respuesta dentro del mismo (posicionamiento, datos biométricos, etc.). La red de sensorización y comunicaciones, debe ser lo suficientemente flexible y escalable, para cubrir todo el escenario de incendio y adaptarse a los cambios que se puedan suscitar.

(ii) Gestión de las comunicaciones: el sistema debe garantizar la disponibilidad de la información en todo momento y lugar dentro de la zona de interés. Debe alcanzar tanto a los recursos desplegados dentro de la zona afectada por el incendio, como a los comandantes y puestos de mando y control fuera de ésta. Una correcta gestión y configuración de los parámetros de calidad de servicio, es fundamental para garantizar la confiabilidad y disponibilidad de la red, considerando que, el sistema tendrá que interactuar y coexistir con otras aplicaciones y sistemas para la gestión de emergencias y desastres, las cuales tienen sus propias demandas de ancho de banda. La interoperabilidad e intercambio de información con otros sistemas y herramientas, es fundamental para promover la coordinación y colaboración entre todos los recursos involucrados en la gestión del GIF.

(iii) Gestión de la información: la información tiene que ser gestionada de manera que se garantice su precisión, inteligibilidad y disponibilidad. Se deben aceptar fuentes y usuarios tanto internos como externos, y la información deberá ser validada previamente, antes de ser puesta a disposición de todos los usuarios. La información de relevancia, debe poder ser almacenada para su reproducción y análisis, tanto durante las operaciones de respuesta como en el futuro, independientemente de su tipo y formato (datos, audio y/o vídeo).

(iv) Herramientas: se pueden clasificar en dos grupos, las de georreferenciación y las de comunicaciones. Dentro de las herramientas de georreferenciación, podemos mencionar las de seguimiento y localización en tiempo real de los recursos desplegados sobre el entorno de operaciones, historial de movimientos, establecimiento de marcas, trazado de perímetros, mapas con información por capas, vistas en 3D, fotografías georreferenciadas, etc. Por otro lado, las herramientas de comunicación, hacen referencia a las facilidades que brinda el sistema para comunicarse e intercambiar información, así como vídeo y audio en tiempo real, envío de alertas, solicitudes de ayuda, mensajería instantánea, etc.

(v) Interfaz hombre-máquina (HMI): aplicación que permite a cualquier usuario del sistema, hacer uso de las herramientas disponibles, de una manera fácil y rápida. El HMI se debe poder instalar y ejecutar tanto en ordenadores de sobre mesa, como en equipos portátiles como tablets, laptops, PDA o smartphones. La aplicación debe ser lo suficientemente intuitiva y promover la usabilidad del sistema, considerando siempre la accesibilidad como un factor crítico dentro de un entorno agresivo como el de las emergencias y desastres. Entre las facilidades que se brindan a los usuarios, podemos mencionar la interfaz gráfica de usuario, botones táctiles, teclas de acceso rápido, mensajes pre-programados, iconos grandes y claros, etc.

(vi) Privacidad y seguridad: serán gestionadas de forma jerárquica, por medio de la asignación de perfiles y permisos a los usuarios registrados, así como con la utilización de mecanismos de encriptación y autenticación como WiFi Protected Access (WPA), WPA2 o Extensible Authentication Protocol (EAP) [13]. Las comunicaciones con otros sistemas y redes externas, se han establecido por medio de protocolos de seguridad como Secure Sockets Layer (SSL), Transport Layer Security (TLS) o Hypertext Transfer Protocol Secure (HTTPS) [14].

En la Fig. 1, se muestra un diagrama de bloques que resume la arquitectura propuesta. 


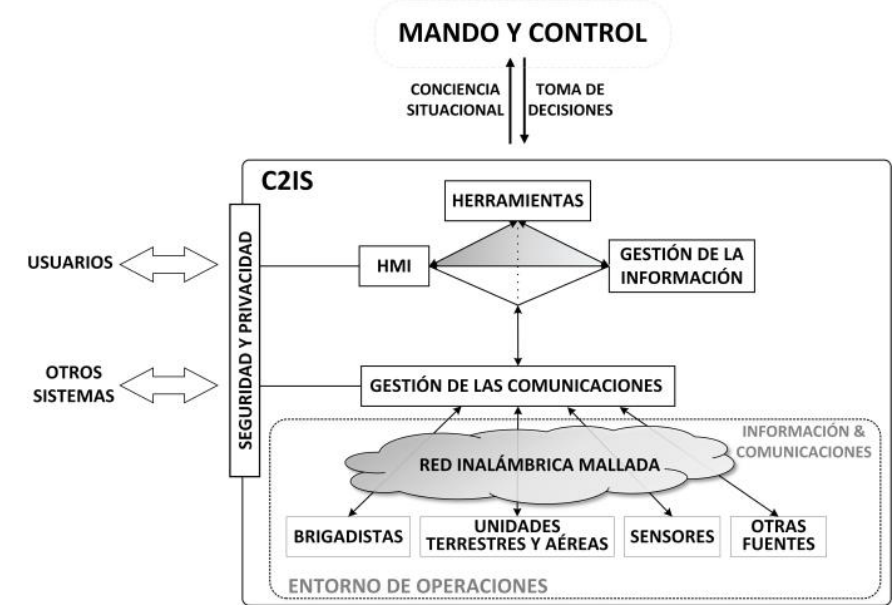

Figura 1. Arquitectura.

La arquitectura ha sido pensada para dar cumplimiento a los requerimientos antes mencionados, y otorgarle agilidad y confiabilidad al sistema. Entre sus características más importantes podemos mencionar:

(i) Una WMN que da soporte a los requerimientos de comunicación, movilidad y accesibilidad de los recursos dentro del entorno de operaciones. Se ha tomado como referencia el estándar IEEE 802.11s, que define las especificaciones de la capa física y enlace de datos para las redes inalámbricas malladas de área local [12] [15]. El estándar establece un Sistema de Distribución Inalámbrico que permite a cada nodo operar como un enrutador dentro de la red. El área afectada, podrá ser cubierta en su totalidad, por medio de múltiples saltos a través de los enlaces establecidos entre los nodos que conforman la red. La existencia de múltiples rutas hacia un mismo destino, otorga confiabilidad y disponibilidad a la arquitectura, y las características de auto-configuración y autoorganización de las WMN, facilitan su implementación y escalabilidad. Entre las responsabilidades de los nodos está el responder a las peticiones de otros nodos, almacenar la información obtenida, y gestionar la operación de la red (flujo de información, establecimiento de nodos coordinadores, seguridad en las comunicaciones, descubrimiento de nuevas rutas, etc.). Los nodos en la arquitectura son identificados como Mesh Points (MP), y aquellos MP que cumplen con las funciones de puntos de acceso a la red para otros nodos, son llamados Mesh Access Points (MAP). Así también, los MP que poseen interfaces hacia otras redes, cumpliendo las funciones de puerta de enlace hacia el exterior, se denominan Mesh Portal Points (MPP) [11]. Además, los MP pueden ser fijos o móviles, aéreos o terrestres, dependiendo de los recursos disponibles y los requerimientos propios de la situación. Cada nodo desplegado dentro del campo de operaciones, deberá estar provisto del equipamiento que permita conocer con exactitud su estado, posición, y colaborar con la descripción del entorno (terminales de comunicación, sensores biométricos y telemétricos, cámara de vídeo, GPS, etc.). En cuanto a la seguridad, se toma como base el estándar 802.11i, utilizando WPA2 como mecanismo para garantizar la seguridad y privacidad de la red [16].

Hay que tener en cuenta, que si bien cada escenario tiene sus propios requerimientos, de acuerdo a las particularidades del campo de operaciones y del GIF, el rendimiento de una WMN siempre está en relación directa con el número de saltos, la distancia entre nodos y la movilidad de los usuarios [12]. Con el objeto de garantizar la operatividad de la red y de acuerdo con los resultados obtenidos en las simulaciones y pruebas de funcionalidad, se estima que cada recurso dentro del campo de operaciones, se ubique a no más de tres saltos del MPP. Así también, se establece en seis, el número máximo de usuarios que pueden ser atendidos por cada MAP, tomando como base un ancho de banda requerido para cada usuario de $500 \mathrm{Kbps}$ para voz, datos y vídeo; y que el rendimiento promedio de un enlace $802.11 \mathrm{~s}$ con tres saltos oscila en el orden de los 2 Mbps [12].

(ii) La gestión de datos se realiza por medio de un mecanismo de réplica en tiempo real que permite la asignación de prioridades y calidad de servicio a la transmisión de datos. Así también, se utiliza un sistema de gestión de base de datos relacional ( $M y S Q L$ Server [17] versión 5.5 en adelante), para garantizar la integridad referencial y evitar la duplicidad de registros.

La gestión de vídeo es uno de los puntos fuertes de esta arquitectura. Se define la implementación de un conjunto jerárquico y distribuido de servidores de vídeo, que optimizan el acceso a las videocámaras de los recursos desplegados sobre el campo de operaciones. Se establece una única conexión con cada videocámara a través del servidor más cercano, y el acceso al vídeo por parte de los usuarios, se hace siempre desde el servidor más cercano, optimizando el uso de ancho de banda y evitando la saturación en los enlaces y las peticiones hacia las videocámaras.

Se pueden implementar tantos servidores como sean necesarios, y los vídeos pueden o no ser recopilados dentro de una unidad de almacenamiento predefinida, en diferentes tipos de formatos como MP4, AVI, MKV, etc.

Se propone UDP/RTP/RTCP [16] como protocolo para la transmisión de vídeo, dando soporte a los requerimientos de tiempo real y variabilidad en los parámetros de comunicación.

(iii) Respecto a la interoperabilidad con otros sistemas y herramientas relacionadas con la gestión de emergencias y desastres, se ha tomado como referencia el Joint Consultation, Command and Control Information Exchange Data Model (JC3IEDM) [18] propuesto por el Programa de Interoperabilidad Multilateral de la OTAN, para el intercambio de información de mando y control en 
ambientes tácticos colaborativos. El intercambio de información entre los sistemas involucrados, se ha estandarizado por medio de HTTP bajo un paradigma RESTful, y un modelo de datos común para toda la información que se requiera compartir [19] [20].

En la Fig. 2, se muestra un ejemplo de una topología acorde con la arquitectura propuesta.



Figura 2. Ejemplo de una posible topología.

\section{VALIDACIÓN Y PRUEBAS DE FUNCIONALIDAD}

La Unión Europea a través de su séptimo programa marco para la investigación e innovación (FP7) [21], desarrolla el proyecto Advanced Forest Fire Fighting (AF3) [22], el cual tiene como objetivo principal, el desarrollo de nuevas tecnologías para mejorar las operaciones de respuesta y gestión de incendios forestales. La Universitat Politècnica de València (UPV) es uno de los colaboradores de AF3, y asiste con el desarrollo del Advanced Situation Awareness System (ASAS), el cual está siendo desarrollado en base a la arquitectura descrita en este documento. El ASAS es el responsable de obtener y distribuir la información para conformar una CS precisa y oportuna del entorno, durante las operaciones de respuesta a un GIF.

La implementación y operatividad de la arquitectura, se validó por medio de pruebas de funcionalidad sobre un prototipo del ASAS, en un escenario simulado para un GIF, en la zona costera de Puig (Valencia, España). Las pruebas consistieron en la supervisión de dos brigadistas $\left(\mathrm{B}_{\mathrm{A}}\right.$ y $\left.\mathrm{B}_{\mathrm{B}}\right)$, y la transmisión de vídeo en tiempo real desde las videocámaras de cada uno de ellos, hasta un puesto de mando y control (CC) ubicado en las instalaciones de la UPV en el exterior del escenario del simulacro (Fig. 3).

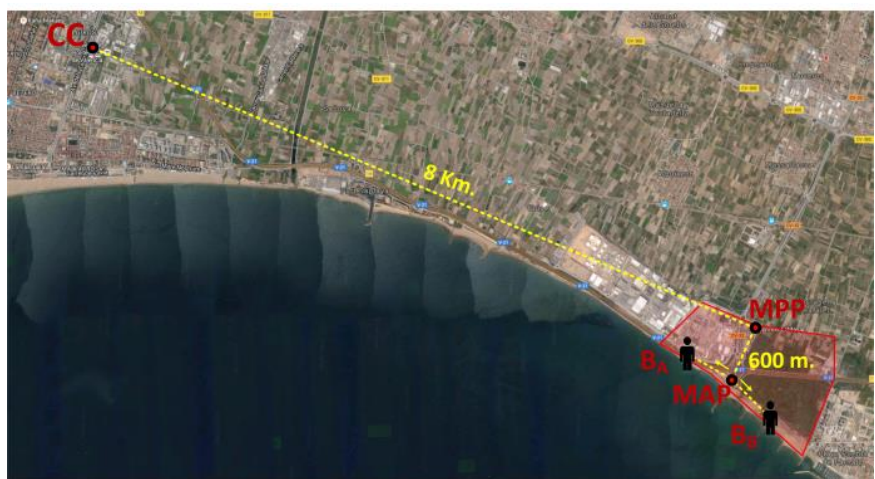

Figura 3. Mapa de la zona de pruebas.
Las pruebas iniciaron con el desplazamiento de los brigadistas a lo largo de la zona del escenario de pruebas, alejándose simultáneamente y en sentido opuesto del MPP. El objetivo fue verificar la efectiva identificación y posicionamiento de los brigadista, y la distancia máxima a la cual se podían transmitir señales de vídeo con una calidad aceptable para describir el entorno de operaciones.

Se implementó una red $802.11 \mathrm{~s} \mathrm{a} 2.4 \mathrm{GHz}$ con cuatro nodos: dos brigadistas dentro del área del simulacro, equipados con radios $802.11 \mathrm{~s}$ (con su correspondiente batería y antena omnidireccional de $13 \mathrm{dBi}$ ), una cámara de vídeo IP, y una PDA rugerizada con GPS y la aplicación ASAS (Fig. 4 y 5); una estación portable 802.11s que desempeñó las funciones de MAP dentro de la zona del incendio; y una estación móvil 802.11s, con un ordenador portable y la aplicación ASAS, que cumplió como MPP, permitiendo la interconexión entre la red táctica y el CC por medio de un enlace WiMax a $2.4 \mathrm{GHz}$ (con antenas direccionales de $16 \mathrm{dBi}$ ).

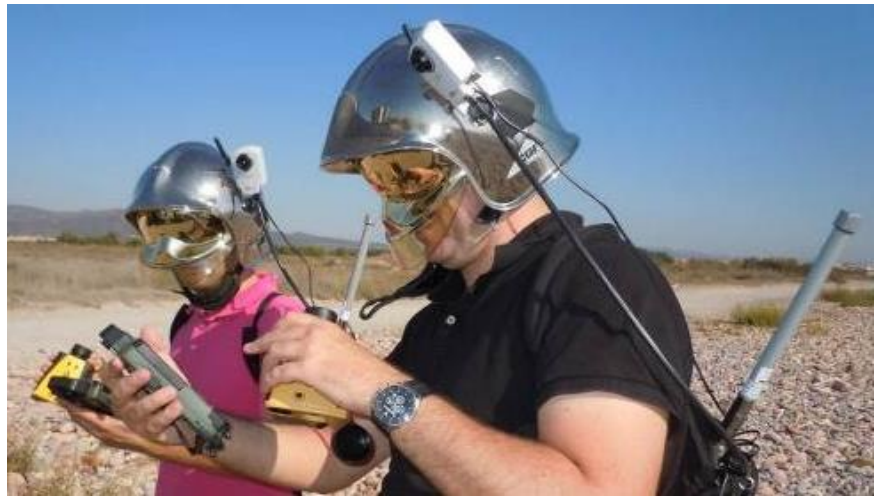

Figura 4. Brigadistas sobre el entorno de pruebas.

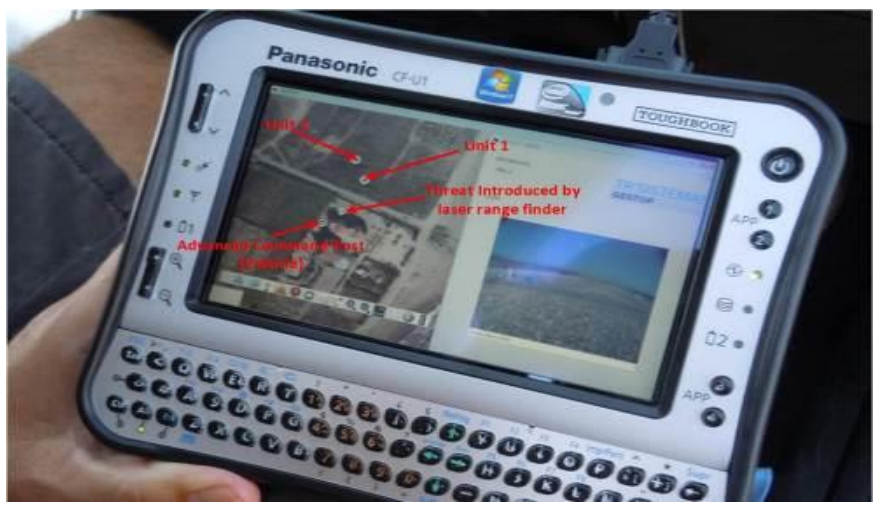

Figura 5. PDA con la aplicación ASAS.

En el MPP se implementó un servidor de vídeo, que permitió el almacenamiento y distribución del vídeo proveniente de las videocámaras de los brigadistas. Se tomó como base un vídeo en formato MPEG4 [23], con 15 tramas por segundo, en un formato estándar de intercambio (CIF, por sus siglas en inglés) con resolución de 352×288 píxeles. El Ancho de banda requerido para la transmisión de una señal con estas características, considerando un factor de movimiento menor de $30 \%$, oscila entre 100 y $500 \mathrm{Kbps}$ [24] [25], por lo que se tomó una tasa de transmisión de $256 \mathrm{Kbps}$ como base para las pruebas de campo. En el CC se implementó un nodo de AF3, que cumplió las funciones de Servidor ASAS y le permitió intercambiar información con otras herramientas de AF3. 


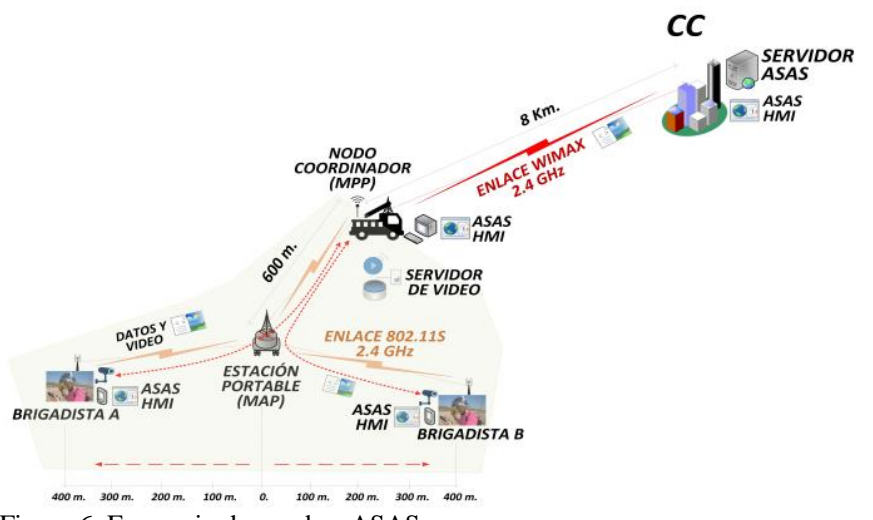

Figura 6. Escenario de pruebas ASAS.

\section{RESUlTADOS}

Las pruebas fueron satisfactorias en cuanto a la factibilidad de implementación y la operatividad del C2IS. La supervisión de los brigadistas dentro del escenario de pruebas se realizó de forma exitosa (Fig. 7). Los datos de identificación y posicionamiento se trasmitieron con una periodicidad de 30 segundos, y dado el tamaño de las peticiones HTTP para esta información (unidades de $\mathrm{KB}$ ), pasaron desapercibidas respecto a los requerimientos de ancho de banda de las señales de vídeo (cientos de KB).

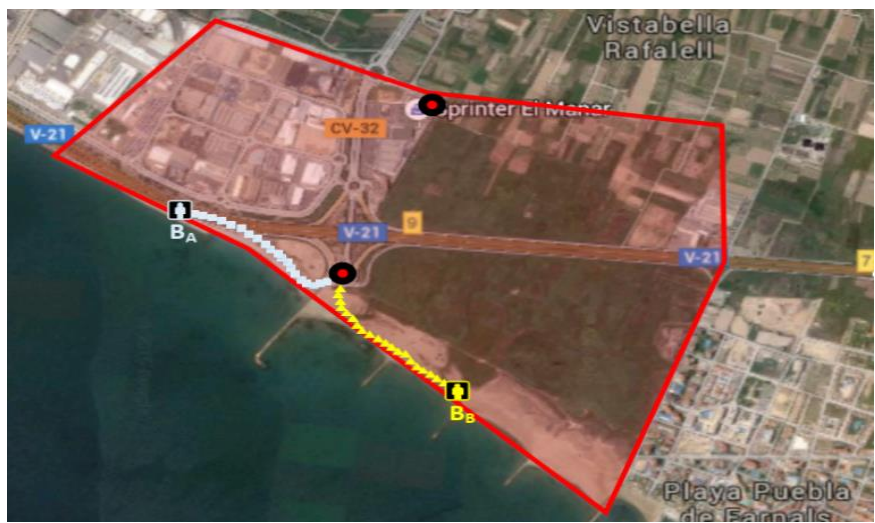

Figura 7. Posicionamiento e historial de seguimiento de los brigadistas.

Las pérdidas y anomalías en los canales de comunicación, fueron aumentando progresivamente en relación directa con el aumento de la distancia entre el MPP y los brigadistas, como se muestra en las Fig. 8.a, 8.b, 8.c, y 8.d. A los 700 m. de distancia entre cada uno de los brigadistas y el MPP (incluidos los 600 $\mathrm{m}$. desde el MAP al MPP), se pudieron observar las primeras anomalías en los canales de comunicación, con pérdidas en las transmisiones inferiores al 1\% (Fig. 8.a), las cuales pasaron prácticamente desapercibidas respecto a la reproducción de los vídeos en el MPP y en el CC. La distancia máxima a la cual se obtuvo una calidad aceptable en la reproducción de vídeo, se determinó en el orden de los 1000 m., con unas pérdidas aproximadas de $20 \%$ en las transmisiones (Fig. 8.d). Para este caso, se pudo observar la existencia de una importante cantidad de ruido en las imágenes y cortes en la reproducción, pero todavía con una calidad aceptable para un entorno de emergencia, es decir, que mediante la reproducción de las señales de vídeo, todavía se pudo obtener información relevante respecto al estado del entorno del simulacro. En el CC, no se observaron variaciones importantes respecto a la calidad de vídeo, puesto que los parámetros de comunicación (ancho de banda, distancia, movilidad, etc.) permanecieron constantes durante las pruebas. Es importante tener en cuenta que las señales de vídeo se distribuyen desde el servidor implementado en el MPP (no desde las cámaras de los brigadistas), y que solamente los brigadistas se encuentran en movimiento, a una distancia administrativa de un salto respecto al MAP y dos saltos respecto al MPP (Fig. 6).

Se utilizó Wireshark [26] como herramienta para capturar el tráfico cursado en la red, y determinar el ancho de banda requerido para cada señal y el porcentaje de paquetes perdidos durante las pruebas.

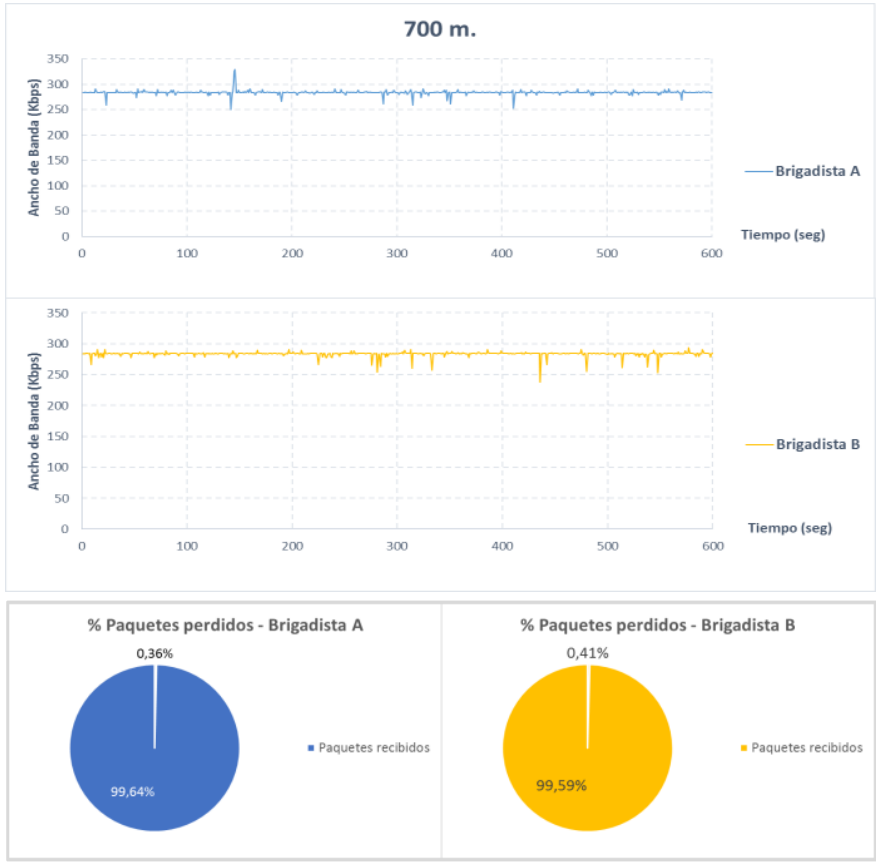

Figura 8.a. Ancho de banda y pérdida de paquetes en las transmisiones a una distancia de $700 \mathrm{~m}$.

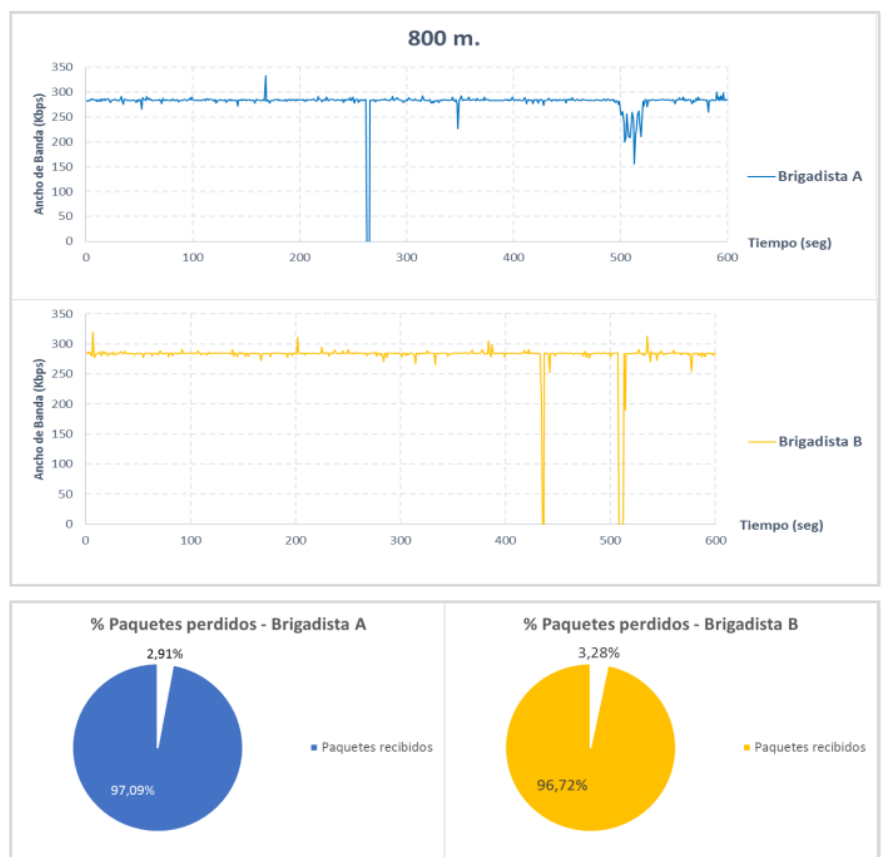

Figura 8.b. Ancho de banda y pérdida de paquetes en las transmisiones a una distancia de $800 \mathrm{~m}$ 




Figura 8.c. Ancho de banda y pérdida de paquetes en las transmisiones a una distancia de $900 \mathrm{~m}$.
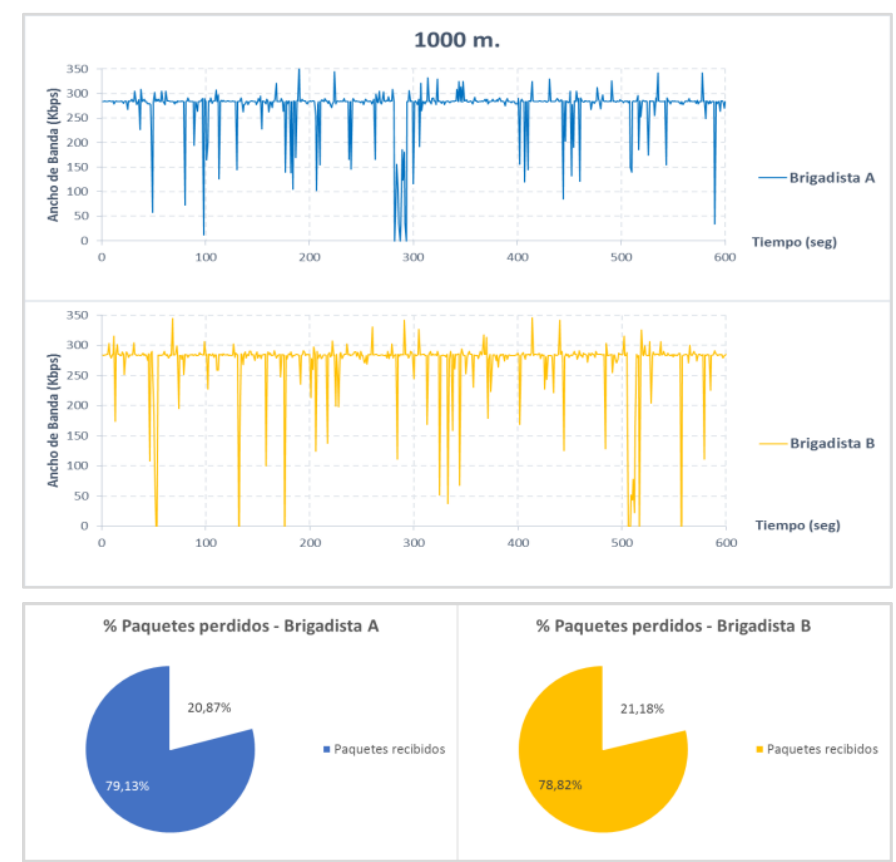

Figura 8.d. Ancho de banda y pérdida de paquetes en las transmisiones a una distancia de $1000 \mathrm{~m}$.

En cuanto al almacenamiento de la información en el MPP, se necesitaron aproximadamente 50MB para cada una de las señales, durante los diez minutos que se tomaron como referencia para las pruebas. Esto implica una capacidad de almacenamiento de 300MB/hora, para cada señal de vídeo que se quiera almacenar.

La información obtenida a través del ASAS, fue tomada por el sistema apoyo a las decisiones y el C2S de AF3, como variables de entrada para la ejecución de los procesos bajo su responsabilidad.

\section{CONCLUSIONES Y NOTAS FINALES}

Este artículo describe una arquitectura para un Sistema de Información de Mando y Control, que posibilita la adquisición de una Conciencia Situacional precisa y común para todos los involucrados, del entorno de operaciones al momento de enfrentar un incendio forestal de grandes magnitudes. La arquitectura se validó por medio de pruebas de funcionalidad sobre un prototipo que permitió, la supervisión de dos brigadistas dentro de un escenario simulado para un incendio, y la descripción de este escenario mediante las transmisiones de vídeo en tiempo real, desde las videocámaras que cada brigadista llevaba consigo. El sistema cumplió a satisfacción con los objetivos propuestos, permitiendo la descripción del entorno de operaciones y la supervisión de las fuerzas de respuesta desplegadas dentro de éste, promoviendo el mando y control, la coordinación y colaboración entre las fuerzas implicadas en las operaciones de respuesta.

Se pudo comprobar la flexibilidad de la arquitectura; tanto para permitir la interacción entre diferentes tecnologías de comunicación (Wimax y WiFi para las pruebas de campo realizadas), como respecto a su interoperabilidad con otros sistemas y herramientas para la gestión de emergencias y desastres. Los requerimientos de servicio, cobertura y movilidad de los usuarios fueron cubiertos satisfactoriamente por la tecnología $802.11 \mathrm{~s}$ utilizada durante las pruebas, tanto para la transmisión de vídeo en tiempo real, como para los datos de posicionamiento de los brigadistas.

Las mediciones se realizaron sobre un escenario que, de manera general, presenta condiciones ambientales semejantes al de un entorno real en el cual se puede presentar un GIF. Sin embargo, se tiene que considerar que en un escenario real, las condiciones siempre son más dinámicas y se pueden presentar situaciones inesperadas mucho menos amigables. Es aquí donde una topología mallada, así como las unidades portables y móviles, presentan sus beneficios reales.

La distancia efectiva entre nodos se podría incrementar utilizando técnicas como Multiple-input Multiple-output (MIMO) [27], antenas direccionales (para nodos que permanezcan fijos) o con mayor ganancia, entre otras alternativas, que en teoría aumentarían el alcance de los enlaces de comunicación, tomando siempre en consideración que con el aumento de la distancia también aumentan las anomalías en los canales de comunicación.

En cuanto a los trabajos futuros, se está desarrollando un aplicativo que permita redundancia en los canales de comunicación, por medio de la utilización simultánea de diferentes tecnologías, y la conmutación automática entre ellas, en base a la mejor calidad de señal percibida.

\section{REFERENCIAS}

[1] WWF/Adena, Bosques vulnerables a grandes incendios, Madrid, 2013.

[2] NPS, «Fire and Aviation Management» National Park Services U.S., June 2016. [En línea]. Available: https://www.nps.gov/fire/wildlandfire/learning-center/fire-in-depth/wildfire-causes.cfm.

[3] Ecovive.com, «Causas de los Incendios Forestales,» June 2016. [En línea]. Available: http://www.ecovive.com/causas-de-los-incendiosforestales.

[4] E. Adam, Fighter cockpits of the future, vol. 12th DASC, IEEE/AIAA Digital Systems Conference, 1993, pp. 318-323. 
[5] J. Guarque, Estrategias y tácticas. Los puntos clave en la gestión de incendios forestales en las WUI, Catalunya: Cos Bombers Generalitat Catalunya, 2014.

[6] C. Cunliffe Checura, Factor Humano - Conciencia Situacional, Fuerza Aérea de Chile - Centro de Medicina Aeroespacial, 2007.

[7] J. Sande Silva, F. Rego, P. Fernandes y E. Rigolot, Towards Integrated Fire Management - Outcomes of the European Project fire Paradox, Porvoo: European Forest Institute, 2010.

[8] A. Vidan y G. Hogan, Integrated Sensing and Command and Control System for Disaster Response, Whaltam: IEEE, 2010, pp. 185-189.

[9] D. S. Alberts y R. E. Hayes, Understanding Command and Control, Washington: CCRP Publication Series, 2006.

[10] National Geographic, «National Geographic - Incendios,» 2016. [En línea]. Available: http://nationalgeographic.es/medioambiente/desastres-naturales/incendios.

[11] Y. Zhang, J. Luo y H. Hu, Wireless Mesh Networking, New York: Auerbach Publications, 2007.

[12] G. Hiertz, D. Denteneer, S. Max, R. Taori, J. Cardona, L. Berlemann y B. Walke, IEEE 802.11S: THE WLAN MESH STANDARD, IEEE Wireless Communications, 2010.

[13] A. H. Adnan, M. Abdirazak, A. S. Sadi, T. Anam, S. Z. Khan, M. M. Rahman y M. M. Omar, A comparative study of WLAN security protocols: WPA, WPA2, Bashundhara: IEEE, 2015, pp. 165-169.

[14] J. Clark y P. C. Oorschot, SoK: SSL and HTTPS: Revisiting Past Challenges and Evaluating Certificate Trust Model Enhancements, San Francisco, California: IEEE, 2013.

[15] H. Jerome y M. Burton, 802.11s Mesh Networking, Certified Wireless Network Professional, 2011.

[16] A. H. Adnan, M. Abdirazak, A. B. M. S. Sadi, T. Anam, S. Z. Khan, M. M. Rahman y M. M. Omar, A comparative study of WLAN security protocols: WPA, WPA2, Dhaka: IEEE, 2015, pp. 165-169.

[17] MySQL AB, «MySQL,» June 2016. [En línea]. Available: https://www.mysql.com/.

[18] MIP OTAN, «Overview of the Joint C3 information exchange data model (JC3IEDM overview),» Febrary 2012. [En línea]. Available: https://mipsite.lsec.dnd.ca/Public\%20Document\%20Library/04Baseline_3.1/Interface-Specification/JC3IEDM/JC3IEDM-Overview3.1.4.pdf.

[19] UCI, «Representational State Tranfer,» June 2016. [En línea]. Available:

https://www.ics.uci.edu/ fielding/pubs/dissertation/rest_arch_style.ht $\mathrm{m}$.

[20] «Introducing JSON,» junio 2016. [En línea]. Available: http://www.json.org/.

[21] European Commission, «Research and innovation FP7,» June 2016. [En línea]. Available: https://ec.europa.eu/research/fp7/index_en.cfm.

[22] European Cimmission, «AF3 - Advanced Forest Fire Fighting,» June 2016. [En línea]. Available: http://af3project.eu/af3/.

[23] D. Marpe, T. Wiegand y G. Sullivan, The H.264/MPEG4 Advanced Video Coding standard and its Applications, IEEE, 2006, pp. 134-143.

[24] Motorola, «Video Solutions: Understanding Video Image Quality,» $2007 . \quad$ [En línea]. Available: http://www.motcom.ch/UserFiles/Video_Quality_White_Paper.pdf.

[25] IBM Knowledge Center, «Managing video bit-rate,» 2016. [En línea]. Available:

http://www.ibm.com/support/knowledgecenter/SSKTXQ_8.5.0/com.i bm.help.sametime.v85.doc/admin/admin_av_video_resolution.html.

[26] «Wireshark,» MAyo 2016. [En línea]. Available: https://www.wireshark.org/.

[27] H. Ezzeldin, R. Hariharan, A. M. A. y K. Dina, A Real-time 802.11 Compatible Distributed MIMO System, London: Associations for Computing Machinery, 2015, pp. 119-120.

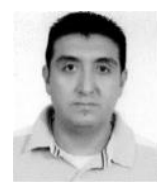

Marcelo Zambrano obtuvo el título de Ingeniero en Electrónica y Telecomunicaciones en 2001, y Magister en Gestión Empresarial en 2009, ambos en la Escuela Politécnica Nacional, Quito-Ecuador. Actualmente se encuentra realizando un Ph.D. en Telecomunicaciones en la Universitat Politècnica de València en España. Más de 15 años de experiencia en empresas relacionadas con el sector de la informática y redes, y su campo de investigación actualmente se centra sobre los sistemas y aplicaciones de tiempo real orientados a la gestión de emergencias.

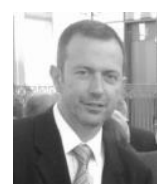

Israel Pérez Llopis obtuvo su maestría en ingeniería informática y su doctorado en telecomunicaciones en la Universitat Politècnica de València, en 2000 y 2009 respectivamente. Es parte del Departamento de Comunicaciones de la UPV desde 2004, donde trabaja como investigador en el Laboratorio de Aplicaciones y Sistemas de Tiempo Real Distribuido. Está activamente involucrado en proyectos nacionales e internacionales de investigación y desarrollo, principalmente con agencias gubernamentales, defensa y la Comisión Europea EU-FP6, FP7-UE y H2020. Sus áreas de interés incluyen los sistemas de tiempo real, sistemas de mando y control y las comunicaciones tácticas.

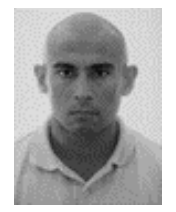

Federico Carvajal se recibió como Ingeniero de Telecomunicación en 1999 y como Ph.D. en el 2007, ambas titulaciones en la Universitat Politècnica de València. Actualmente trabaja en el Laboratorio de Aplicaciones y Sistemas de Tiempo Real Distribuido como investigador principal y director de proyectos. Ha estado involucrado en la realización y gestión de varios proyectos europeos. Posee amplia experiencia en sistemas de mando y control, comunicaciones tácticas y actividades de elearning.

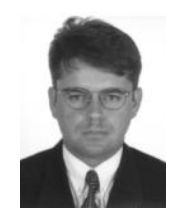

Manuel Esteve Domingo obtuvo tanto su M.Sc. en ingeniería informática como su Ph.D. en telecomunicaciones (Dr.Ing.) en la Universitat Politècnica de València, en 1989 y 1994 respectivamente. Profesor titular en la Escuela Técnica Superior de Ingenieros de Telecomunicaciones (UPVLC) y dirige el grupo de investigación de Sistemas y Aplicaciones de Tiempo Real Distribuido. Cuenta con más de 20 años de experiencia en la investigación de áreas relacionadas con las TICs y networking. Actualmente dirige varios proyectos de $\mathrm{I}+\mathrm{D}$ de índole regional, nacional e internacional y ha colaborado en el desarrollo de proyectos para las agencias gubernamentales, defensa, FP7 y H2020 de la UE. Es autor y coautor de más de 120 trabajos de investigación.

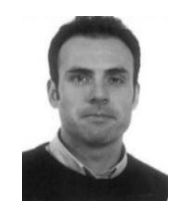

Carlos E. Palau obtuvo su M.Sc. y Ph.D. (Dr.Ing.) en ingeniería de telecomunicaciones, ambos en la Universitat Politècnica de València, en 1993 y 1997 respectivamente. Actualmente es Profesor Titular en la Escuela Técnica Superior de Ingenieros de Telecomunicación y cuenta con más de 18 años de experiencia en el área de investigación de TICs y Networking. Ha colaborado en la I+D de proyectos de streaming multimedia, seguridad, redes y comunicaciones inalámbricas para agencias gubernamentales, defensa y Comisión Europea. Es autor y coautor de más de 120 trabajos de investigación y miembro del TPC de varias conferencias IEEE, ACM e IFIP. 
